\title{
Conjugate Gradient Methods for Computing Weighted Analytic Center for Linear Matrix Inequalities Using Exact and Quadratic Interpolation Line Searches
}

\author{
Shafiu Jibrin *, Ibrahim Abdullahi \\ Department of Mathematics, Faculty of Science, Federal University, Dutse, Jigawa State, Nigeria \\ Email address: \\ shafiuj@yahoo.com (S. Jibrin), Ibrahim.abdullahi@fud.edu.ng (I. Abdullahi) \\ ${ }^{*}$ Corresponding author
}

\section{To cite this article:}

Shafiu Jibrin, Ibrahim Abdullahi. Conjugate Gradient Methods for Computing Weighted Analytic Center for Linear Matrix Inequalities Using Exact and Quadratic Interpolation Line Searches. American Journal of Applied Mathematics. Vol. 8, No. 1, 2020, pp. 1-10. doi: 10.11648/j.ajam.20200801.11

Received: October 18, 2019; Accepted: November 27, 2019; Published: January 4, 2019

\begin{abstract}
We study the problem of computing the weighted analytic center for linear matrix inequality constraints. In this paper, we apply conjugate gradient (CG) methods to find the weighted analytic center. CG methods have low memory requirements and strong local and global convergence properties. The methods considered are the classical methods by Hestenes-Stiefel (HS), Fletcher and Reeves (FR), Polak and Ribiere (PR) and a relatively new method by Rivaie, Abashar, Mustafa and Ismail (RAMI). We compare performance of each method on random test problems by observing the number of iterations and time required by the method to find the weighted analytic center for each test problem. We use Newton's method exact line search and Quadratic Interpolation inexact line search. Our numerical results show that PR is the best method, followed by HS, then RAMI, and then FR. However, PR and HS performed about the same with exact line search. The results also indicate that both line searches work well, but exact line search handles weights better than the inexact line search when some weight is relatively much larger than the other weights. We also find from our results that with Quadratic interpolation line search, FR is more susceptible to jamming phenomenon than both PR and HS.
\end{abstract}

Keywords: Linear Matrix Inequalities, Weighted Analytic Center, Semidefinite Programming, Conjugate Gradient Methods

\section{Introduction}

We consider the following system of linear matrix inequality constraints:

$$
A^{(j)}(x):=A_{0}^{(j)}+\sum_{i=1}^{n} x_{i} A_{i}^{(j)} \succeq 0,(j=1,2, \ldots, q)
$$

where $x \in \mathrm{IR}^{n}$ is a variable and each $A_{i}^{(j)}$ is $m_{j} \mathrm{x} m_{j}$ symmetric matrix.

LMI constraints have applications in engineering, geometry, statistics and in the field of semidefinite programming ([1-6]). Let $\mathcal{R}$ denote the feasible region defined by the inequalities (1). We will assume that the feasible region $\mathcal{R}$ is bounded and it has a nonempty interior.

Given $\omega>0$, the weighted analytic centered for the system
(1) is optimal solution of the optimization problem ([7-9]):

$$
\min \left\{\varphi_{\omega}(x) \mid x \in \mathrm{IR}^{n}\right\}
$$

where,

$$
\phi_{\omega}(x)= \begin{cases}\sum_{j=1}^{q} \omega_{j} \log \operatorname{det}\left[\left(A^{(j)}(x)\right)^{-1}\right] & \text { if } x \in \operatorname{int}(\mathcal{R}) \\ \infty & \text { otherwise }\end{cases}
$$

In the special case of linear constraints, weighted analytic center has been studied extensively in the past (for example, [10]). A weighted analytic center for LMIs which extends the definition given in [10] was given in [7,8].

An infeasible start Newton's method for computing the weighted analytic center was presented in $[11,12]$ and in the case of a single LMI constraint in [13]. The standard Newton's 
method can also be used to compute the weighted analytic center given a starting interior point [7]. Newton's method requires the gradient and Hessian of the objective function $\varphi_{\omega}(x)$ or the Jacobian of a residual function. In this paper, we give conjugate gradient (CG) methods for finding the weighted analytic center starting from a given interior point. CG methods use only the gradient of $\varphi_{\omega}(x)$ and do not require the Hessian of $\varphi_{\omega}(x)$. This approach is particularly beneficial when the dimensions $m_{j}$ of the matrices are high. CG methods also have low memory requirements and strong local and global convergence properties [14].

In this work, we focus on four conjugate gradient methods The methods considered are the classical methods by Hestenes-Stiefel (HS), Fletcher and Reeves (FR), Polak and Ribiere (PR) discussed in [14] and a relatively new method by Rivaie, Abashar, Mustafa and Ismail (RAMI) [15]. We compare performance of each method on random test problems by observing the number of iterations and time required by the method to find the weighted analytic center for each test problem. We use Newton's method exact line search and Quadratic Interpolation inexact line search.

\section{Conjugate Gradient Methods}

Consider a continuously differentiable function $f: \mathrm{R}^{\mathrm{n}} \rightarrow \mathrm{IR}$ and the following unconstrained optimization problem

$$
\min \left\{f(x): x \in \mathrm{R}^{\mathrm{n}}\right\} .
$$

Let $g(x)$ denote the gradient of $f(x)$. A conjugate gradent method to find a solution to problem 3 works as follows. Given an initial guess $x_{o} \in \mathrm{R}^{\mathrm{n}}$, a sequence $\left\{x_{k}\right\}$ is generated by:

$$
x_{k+1}=x_{k}+\alpha_{k} d_{k}
$$

and the direction $d_{k}$ is defined as

$$
d_{k+1}= \begin{cases}-g_{k} & \text { if } k=0 \\ -g_{k+1}+\beta_{k} d_{k} & \text { if } k \geq 1\end{cases}
$$

where $x_{k}$ is the current iterate, $g_{k}=g\left(x_{k}\right), \beta_{k}$ is the CG coefficient and $\alpha_{k}>0$ is the step-length obtained by a line search. A common convergence criterion for a CG method is $\|g(x k)\| \leq T O L$, where $T O L$ is a given tolerance.

Over the years, a variety of CG formulas were given, where majorly, differences are in the parameter $\beta_{k}$. The work by [13] discussed details on some CG methods with special emphasis on their global convergence. In recent times, research carried out by [16-23, 15] focused on some modified CG methods. The summary of the CG methods considered in this work are given in the Table 1, where $\|$. denotes the Euclidean norm.

Table 1. The classical formulas for parameter $\beta_{k}$.

\begin{tabular}{llll}
\hline No. & $\boldsymbol{\beta}_{k}$ & Method name & References \\
\hline 1 & $\frac{\left\|g_{k+1}\right\|^{2}}{\left\|g_{k}\right\|^{2}}$ & Fletcher-Reeves (FR) method & {$[24]$} \\
2 & $\frac{g_{k+1}{ }^{T} y_{k}}{\left\|g_{k}\right\|^{2}}$ & Polak-Rebiere-Polyak (PR) method & {$[25]$} \\
3 & $\frac{g_{k+1}{ }^{T} y_{k}}{d_{k} y_{k}}$ & Hestenes-Stiefel (HS) method & {$[26]$} \\
4 & $\frac{g_{k+1}{ }^{T}\left(g_{k+1}-\frac{\left\|g_{k+1}\right\|}{\left\|g_{k}\right\|} \mid g_{k}\right.}{d_{k}{ }^{T}\left(d_{k-} g_{k+1}\right)}$ & (RAMI) method & {$[15]$} \\
\hline
\end{tabular}

For convex quadratic problems, the first three methods in Table 1 are equivalent using exact line search to compute the step length $\alpha$, but behave differently if the objective function $f(x)$ is non-convex. The classical method FR possess strong convergence properties but not computationally powerful. While, methods like PR and HS perform better computationally, but may not always converge. Problems associated with classical methods gave room for improvement through modification and hybridization. Convergence analysis and numerical experiments showed that RAMI method proposed by [15] is robust as compared to FR and PR, since it solved all the benchmark problems under consideration, while FR and PR did not.

In this research, these four CG methods are employed to find weighted analytic centers for the system (1), observe and compare their computational strengths.

The gradient $g$ of the barrier function $\varphi_{\omega}(x)$ is given by [8]:

$g_{i}(x)=\nabla_{i} \phi_{\omega}(x)=-\sum_{j=1}^{q} \omega_{j}\left(A^{(j)}(x)\right)^{-1} ? A_{i}^{(j)},(i=1, \ldots, n)$

The Hessian $H(x)$ of $\varphi_{\omega}(x)$ is given by [8]:

$$
H_{i j}(x)=\sum_{k=1}^{q} \omega_{k}\left[\left(A^{(k)}(x)\right)^{-1} A_{i}^{(k)}\right]^{T} ?\left[\left(A^{(k)}(x)\right)^{-1} A_{j}^{(k)}\right],(i, j=1, \ldots, n)
$$

\section{Line Searches for Our Conjugate Gradient Methods}

We find exact and inexact step-sizes for our conjugate gradient methods. Newton's method is used to find the exact step-size and inexact step-size is found using Quadratic interpolation. We also discuss convergence for the methods.

Let $x$ be an interior point of $\mathcal{R}$, then $A^{(j)}(x) \succ 0$ for each constraint $j$, and the square root of $A^{(j)}(x)$ exists. Given a search direction vector $d$, define the symmetric matrix at $x$ 


$$
B_{j}(d, x)=-A^{(j)}(x)^{-\frac{1}{2}}\left(\sum_{i=1}^{n} d_{i} A_{i}^{(j)}\right) A^{(j)}(x)^{-\frac{1}{2}},(1 \leq j \leq q)
$$

Consider the objective barrier function $\varphi_{\omega}(x)$. Let $d_{k}$ be a conjugate direction generated by the $\mathrm{CG}$ algorithm at the current iterate $x_{k}$. Let $h(\alpha)=\varphi_{\omega}\left(x_{k}+\alpha d_{k}\right)$. The exact step-size $\alpha_{k}$ is given by

$$
\alpha_{k}=\operatorname{argmin}\{h(\alpha) \mid \alpha \geq 0\} .
$$

The following results reduces the cost of computing the exact stepsize using Newton's method.

Theorem 1 Let $x_{k}$ be an interior point of $\mathcal{R}$ and $\lambda_{i}^{(j)}$ be the ith eigenvalue of $B_{j}\left(d_{k}, x_{k}\right)$. Then

$$
h(\alpha)=-\sum_{j=1}^{q} \omega_{j} \log \operatorname{det}\left(A^{(j)}\left(x_{k}\right)\right)-\sum_{j=1}^{q} \omega_{j} \sum_{i=1}^{m_{j}} \log \left(1+\alpha \lambda_{i}^{(j)}\right)
$$

Proof:

$$
\begin{aligned}
& h(\alpha)=\phi_{w}\left(x_{k}+\alpha d_{k}\right) \\
& =\sum_{j=1}^{q} w_{j} \log \operatorname{det}\left[\left(A^{(j)}\left(x_{k}+\alpha d_{k}\right)\right)^{-1}\right] \\
& =-\sum_{j=1}^{q} w_{j} \log \operatorname{det}\left[A^{(j)}\left(x_{k}+\alpha d_{k}\right)\right] \\
& =-\sum_{j=1}^{q} w_{j} \log \operatorname{det}\left[A_{0}^{(j)}+\sum_{i=1}^{m_{j}}\left(x_{k}+\alpha d_{k}\right)_{i} A_{i}^{(j)}\right] \\
& =-\sum_{j=1}^{q} w_{j} \log \operatorname{det}\left[A_{0}^{(j)}+\sum_{i=1}^{m_{j}}\left(x_{k}\right)_{i} A_{i}^{(j)}+\sum_{i=1}^{m_{j}} \alpha\left(d_{k}\right)_{i} A_{i}^{(j)}\right] \\
& =-\sum_{j=1}^{q} w_{j} \log \operatorname{det}\left[A^{(j)}\left(x_{k}\right)+\alpha B_{j}\left(d_{k}, x_{k}\right)\right] \\
& \begin{array}{l}
=-\sum_{j=1}^{q} w_{j} \log \operatorname{det}\left[A^{(j)}\left(x_{k}\right)^{\frac{1}{2}}(I+\right. \\
\left.\left.\alpha A^{(j)}\left(x_{k}\right)^{-\frac{1}{2}} B_{j}\left(d_{k}, x_{k}\right) A^{(j)}\left(x_{k}\right)^{-\frac{1}{2}}\right) A^{(j)}\left(x_{k}\right)^{\frac{1}{2}}\right]
\end{array}
\end{aligned}
$$

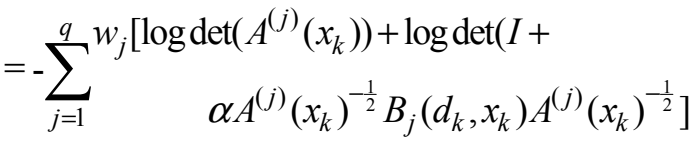

$$
\begin{aligned}
& =-\sum_{j=1}^{q} w_{j}\left[\log \operatorname{det}\left(A^{(j)}\left(x_{k}\right)\right)+\sum_{i=1}^{m_{j}}\left(1+\alpha \lambda_{i}^{(j)}\right)\right] \\
& \left.=-\sum_{j=1}^{q} w_{j} \log \operatorname{det}\left(A^{(j)}\left(x_{k}\right)\right)-\sum_{j=1}^{q} w_{j} \sum_{i=1}^{m_{j}} \log \left(1+\alpha \lambda_{i}^{(j)}\right)\right]
\end{aligned}
$$

Corollary 1 The derivatives of $h(\alpha)$ are given by

$$
\begin{aligned}
& h^{\prime}(\alpha)=-\sum_{j=1}^{q} w_{j} \sum_{i=1}^{m_{j}} \frac{\lambda_{i}^{(j)}}{\left(1+\alpha \lambda_{i}^{(j)}\right)} \\
& h^{\prime \prime}(\alpha)=\sum_{j=1}^{q} w_{j} \sum_{i=1}^{m_{j}}\left(\frac{\lambda_{i}^{(j)}}{1+\alpha \lambda_{i}^{(j)}}\right)^{2}
\end{aligned}
$$

Exact line search is applied to compute the step-size $\alpha_{k}(8)$ in our CG algorithms using one-dimensional Newton's method starting from $\alpha=0$ :

$$
\alpha_{k+1}=\alpha_{k}-\frac{h^{\prime}\left(\alpha_{k}\right)}{h^{\prime \prime}\left(\alpha_{k}\right)}
$$

We will need the following result to approximate the step-size $\alpha_{k}(8)$ using Quadratic interpolation. The proof of Theorem 2 can be found in [27].

Denote the largest positive eigenvalue of a symmetric matrix $B$ by $\lambda^{+}{ }_{\max }(B)$.

Theorem 2 Let $\mathrm{x}_{\mathrm{k}}$ be an interior point of $\mathcal{R}$. Assume the ray $\left\{\mathrm{x}_{\mathrm{k}}+\sigma \mathrm{d}_{\mathrm{k}} \mid \sigma \geq 0\right\}$ intersects the boundary of $A^{(j)}(x) \succeq 0$ at the point $x_{k}+\sigma_{+}^{(j)} d_{k}$. Then, the distance to the boundary along the ray from $x_{k}$ is given by

$$
\sigma_{+}^{(j)}=1 / \lambda_{\max }^{+}\left(B_{j}\left(d_{k}, x_{k}\right)\right)
$$

Proof: $A^{(j)}(x) \succ 0$ when $x$ is in the interior of $\mathcal{R}$, but on the boundary it must have at least one zero eigenvalue. Then

$$
\sigma_{+}^{(j)}=\min \left\{\mu \mid \mu>0, \operatorname{det}\left[A^{(j)}\left(x_{k}+\mu d_{k}\right)\right]=0\right\}
$$

Now, when $\mu>0$

$$
\begin{gathered}
\operatorname{det}\left[A^{(j)}\left(x_{k}+\mu d_{k}\right)\right]=0 \Leftrightarrow \operatorname{det}\left[A^{(j)}\left(x_{k}\right)+\mu \sum_{i=1}^{n}\left(d_{k}\right)_{i} A_{i}^{(j)}\right]=0 \\
\Leftrightarrow \operatorname{det}\left[\frac{1}{\mu} I+A^{(j)}\left(x_{k}\right)^{-\frac{1}{2}}\left(\sum_{i=1}^{n}\left(d_{k}\right)_{i} A_{i}^{(j)}\left(x_{k}\right)^{-\frac{1}{2}}\right]=0\right. \\
\Leftrightarrow \operatorname{det}\left[\frac{1}{\mu} I-B_{j}\left(d_{k}, x_{k}\right)\right]=0 \\
\Leftrightarrow \frac{1}{\mu} \text { is an eigenvalue of } B_{j}\left(d_{k}, x_{k}\right) \\
\Leftrightarrow \mu \text { is an eigenvalue of } B_{j}\left(d_{k}, x_{k}\right)^{-1}
\end{gathered}
$$

This and (14) give

$$
\sigma_{+}^{(j)}=1 / \lambda_{\max }^{+}\left(B_{j}\left(d_{k}, x_{k}\right)\right)
$$

The distance $\sigma_{+}$from $x_{k}$ to the boundary of the bounded feasible region $\mathcal{R}$ in the direction $d_{k}$ is given by 


$$
\sigma_{+}=\min \left\{\sigma_{+}^{(j)} \mid 1 \leq j \leq q\right\}
$$

where $\sigma_{+}^{(j)}$ is given by (13). Note that $\sigma_{+}$exists since $\mathcal{R}$ is bounded and $x_{k}$ is an interior point of $\mathcal{R}$.

The following describes Quadratic interpolation line search for approximating the step-sze $\alpha_{k}(8)$ in our CG algorithms (see [28]).

Quadratic Interpolation

Step 1: Use (15) to find the distance $\sigma_{+}$from $x_{k}$ to the boundary of the bounded feasible region $\mathcal{R}$ in the direction $d_{k}$

Step 2: Set $\alpha_{1}=0$ and $\alpha_{3}=\sigma_{+}$

Step 3: Consider $h(\alpha)=\varphi_{\omega}\left(x_{k}+\alpha d_{k}\right)$

Repeat

$$
\alpha_{3}=\alpha_{3} / 2
$$

$$
\text { Until } h\left(\alpha_{3}\right)<h\left(\alpha_{1}\right)
$$

Let $\alpha_{2}=\alpha_{3} / 2$

Step 4: Compute the zero of the quadratic polynomial $P(\alpha)$ passing through the points $\left(\alpha_{1}, P\left(\alpha_{1}\right)\right),\left(\alpha_{2}, P\left(\alpha_{2}\right)\right)$ and $\left(\alpha_{3}\right.$, $\left.P\left(\alpha_{3}\right)\right)$. The zero is given by

$$
\alpha^{*}=\frac{1}{2}\left(\alpha_{2}-\frac{h_{1}}{h_{3}}\right)
$$

where,

$$
\begin{aligned}
& h_{1}=\frac{h\left(\alpha_{2}\right)-h\left(\alpha_{1}\right)}{\alpha_{2}-\alpha_{1}} \\
& h_{2}=\frac{h\left(\alpha_{3}\right)-h\left(\alpha_{2}\right)}{\alpha_{3}-\alpha_{2}} \\
& h_{3}=\frac{h_{2}-h_{1}}{\alpha_{3}-\alpha_{1}} .
\end{aligned}
$$

Step 5:

If $\alpha_{3}<\alpha^{*}$

set $\alpha_{k}=\alpha_{3}$

else

set $\alpha_{k}=\alpha^{*}$

end

Let $x_{0}$ be a starting point for the CG method and consider the level set

$$
\mathcal{L}=\left\{x \in \operatorname{IR}^{n} \mid g(x) \leq g\left(x_{0}\right)\right\}
$$

The gradient $g(x)=\nabla \varphi_{\omega}(x)$ is called Lipschitz continuous in a neighborhood $\mathcal{N}$ of $\mathcal{L}$ if there exists $K \geq 0$ such that

$$
\|g(x)-g(y) \leq K\| x-y \| \forall x, y \in \mathcal{N} .
$$

$\mathcal{L}$ is called bounded if there is $r>0$ such that $\|x\| \leq r \quad \forall x \in \mathcal{L}$.

Let $x_{k}$ be the sequence generated by the CG method. The method is called globally convergent if $\left\|g\left(x_{k}\right)\right\|=0$ for some $k$ or $\liminf _{\mathrm{k} \rightarrow 0} x_{k}=0$.
If the gradient $g(x)=\nabla \varphi_{\omega}(x)$ is Lipschitz continuous in a neighborhood of $\mathcal{L}$, FR method with exact line search is globally convergent [29]. We are not aware of any implementation or convergence analysis for FR with Quadratic interpolation inexact line search in the literature. FR is known to be susceptible to jamming phenomenon where it takes many short steps without significant decrease in the objective function $\varphi_{\omega}(x)[14]$.

$\mathrm{PR}$ is globally convergent when $\varphi_{\omega}(x)$ is strongly convex and the line search is exact [30]. PR and HS method with exact line search coincide and each is globally convergent if $x_{k+1}-x_{k}$ converges to 0 and $g$ is Lipschitz continuous in a neighborhood of $\mathcal{L}$ [31]. Again, we have not seen any implementation or convergence analysis for either PR or HS with Quadratic interpolation inexact line search in the literature. Both PR and HS are less susceptible to jamming phenomenon than FR [14].

RAMI conjugate method with exact line search is globally convergent if $g$ is Lipschitz continuous in a neighborhood of $\mathcal{L}$ and $\mathcal{L}$ is bounded [15]. We are not aware of any implementation or convergence analysis for RAMI with Quadratic interpolation inexact line search in the literature.

The level set $\mathcal{L}$ is bounded since $\mathcal{L} \subseteq \mathcal{R}$ and $\mathcal{R}$ is bounded. Theorem 3 and Theorem 4 show that the conjugate gradient methods with exact line search applied to our weighted analytic center problem are globally convergent. They show that the methods are suitable.

Theorem 3 The barrier function $\varphi_{\omega}(\mathrm{x})$ is strongly convex over the interior of the feasible region $\mathcal{R}$.

Proof: The assumption that $\mathcal{R}$ is bounded and it has a nonempty interior implies that the function $\varphi_{\omega}(\mathrm{x})$ is strictly convex over $\mathcal{R}$ [8]. Hence, the Hessian $\mathrm{H}(\mathrm{x})(2)$ of $\varphi_{\omega}(\mathrm{x})$ has positive eigenvalues in the interior of $\mathcal{R}$. Since $\mathcal{R}$ is bounded, the smallest eigenvalue must have a positive minimum value $\gamma$. So, $H(x) \succeq \gamma I$ in the interior of $\mathcal{R}$. This and the fact that $\varphi_{\omega}(\mathrm{x})$ is twice differentiable ([27]) means that $\varphi_{\omega}(\mathrm{x})$ is strongly convex in the interior of $\mathcal{R}$.

Theorem 4 The gradient $\mathrm{g}(\mathrm{x})=\nabla \varphi_{\omega}(\mathrm{x})$ is Lipschitz continuous in a neighborhood of the level set $\mathcal{L}=\left\{\mathrm{x} \in \mathrm{IR}^{\mathrm{n}} \mid\right.$ $\left.\mathrm{g}(\mathrm{x}) \leq \mathrm{g}\left(\mathrm{x}_{0}\right)\right\}$.

Proof: By [27], $\varphi_{\omega}(\mathrm{x})$ is analytic in the interior of $\mathcal{R}$. So, $\mathrm{g}(\mathrm{x})=\nabla \varphi_{\omega}(\mathrm{x})$ is also analytic in the interior of $\mathcal{R}$. Choose any neighborhood $\mathcal{N}$ of $\mathcal{L} . \mathcal{N}$ is bounded since $\mathcal{N} \subseteq \mathcal{L} \subseteq \mathcal{R}$ and $\mathcal{R}$ is bounded. Let $\mathrm{f}(\mathrm{t})=\mathrm{g}((1-\mathrm{t}) \mathrm{x}+\mathrm{ty}$. By the mean value theorem, for some $\mathrm{c} \in(0,1)$

$$
\begin{aligned}
& \mathrm{g}(\mathrm{y})-\mathrm{g}(\mathrm{x})=\mathrm{f}(1)-\mathrm{f}(0)=\mathrm{f}^{0}(\mathrm{c})=\nabla \mathrm{g}((1-\mathrm{c}) \mathrm{x}+\mathrm{cy}) \cdot(\mathrm{y}-\mathrm{x}) \\
& \text { By Cauchy-Schwartz's inequality, }
\end{aligned}
$$

$$
\begin{aligned}
\mid g(y)-g(x) & |=| \nabla g((1-c) x+c y)(y-x) \mid \\
& \leq\|\nabla g((1-c) x+c y)\|\|y-x\| .
\end{aligned}
$$

Since $\mathcal{N}$ is bounded and $\nabla g$ is continuous on $\mathcal{N}$, there exists $K \geq 0$ such that

$$
|g(y)-g(x)| \leq\|y-x\| \forall x, y \in \mathcal{N}
$$


Hence, $g(x)$ is Lipschitz continuous in $\mathcal{N}$.

\section{Numerical Experiments}

In this section, we present numerical experiments to compare HS, FR, HR and RAMI conjugate gradient methods using exact and interpolation line searches.

All numerical experiments were done using a Lenevo-PC computer with codes written in MATLAB version 8 . In all of the problems, each LMI $A_{0}^{(j)}+\sum_{i=1}^{n} x_{i} A_{i}^{(j)} \succeq 0$ was generated as follows: $A_{0}^{(j)}$ is an $m_{j} \times m_{j}$ diagonal matrix with each diagonal entry chosen from $U(0,1)$ distribution. Each $A_{i}^{(j)} \quad(1 \leq i \leq n)$ is a random $m_{j} \times m_{j}$ symmetric sparse matrix with approximately $0.8 * m^{2}$ nonzero entries generated using the Matlab command $\operatorname{sprandsym}\left(m_{j}, 0.8\right)$. This ensures that each of our test problems is random, and that the origin is an interior point for each test problem. HS, FR, PR and RAMI congujate gradient methods were applied to each problem using a maximum of 1000 iterations and a tolerance $T O L=10^{-4}$. Each method is stopped after 1000 iteration or if $\left\|g\left(x_{k}\right)\right\| \leq T O L$.

Table 2 gives the list of test problems. The second column of the table is ambient dimension $n$ and the third column gives the number $q$ of LMI constraints. The sizes $\left[m_{1}, \ldots, m_{q}\right]$ of the matrices is given in the fourth column.

Table 3 gives the number of iteration and time (in seconds) taken by each method to find the weighted analytic center for the given weights using exact line search. The exact line search was done using one-dimensional Newton's method.

Table 2. Test Problems.

\begin{tabular}{|c|c|c|c|}
\hline LMI Test Problem & $n$ & $q$ & {$\left[m_{1}, \ldots, m_{q}\right]$} \\
\hline 1 & 2 & 2 & {$[1,2]$} \\
\hline 2 & 2 & 3 & {$[5,4,5]$} \\
\hline 3 & 2 & 8 & {$[2,4,5,5,5,1,5,4]$} \\
\hline 4 & 3 & 2 & {$[5,4]$} \\
\hline 5 & 3 & 2 & {$[3,4]$} \\
\hline 6 & 4 & 10 & {$[4,5,1,4,2,3,5,5,2,1]$} \\
\hline 7 & 4 & 7 & {$[2,4,4,5,4,2,1]$} \\
\hline 8 & 5 & 6 & {$[5,1,4,4,4,5]$} \\
\hline 9 & 5 & 4 & {$[4,1,5,1]$} \\
\hline 10 & 6 & 3 & {$[4,1,5]$} \\
\hline 11 & 6 & 8 & {$[2,5,2,5,5,3,5,2]$} \\
\hline 12 & 7 & 2 & $[5,4]]$ \\
\hline 13 & 7 & 4 & {$[1,4,1,2]$} \\
\hline 14 & 8 & 5 & {$[1,1,4,3,3]$} \\
\hline 15 & 8 & 5 & {$[5,4,5,2,5]$} \\
\hline 16 & 9 & 3 & {$[3,2,5]$} \\
\hline 17 & 9 & 3 & {$[5,4,4]$} \\
\hline 18 & 10 & 8 & {$[4,2,3,4,5,4,4,2]$} \\
\hline 19 & 10 & 8 & {$[4,5,3,5,4,2,2,4]$} \\
\hline 20 & 10 & 9 & {$[5,2,5,3,2,1,3,2,2]$} \\
\hline 21 & 3 & 6 & {$[3,4,1,5,4,1]$} \\
\hline 22 & 5 & 7 & {$[2,3,5,5,2,4,2]$} \\
\hline 23 & 5 & 3 & {$[5,5,2]$} \\
\hline 24 & 5 & 9 & {$[2,4,4,1,4,5,3,5,1]$} \\
\hline 25 & 10 & 3 & {$[1,5,2]$} \\
\hline 26 & 5 & 10 & {$[3,4,1,3,1,4,4,5,4,4]$} \\
\hline 27 & 3 & 7 & {$[2,3,4,5,4,1,5]$} \\
\hline 28 & 5 & 7 & {$[2,3,5,5,2,4,2]$} \\
\hline 29 & 3 & 8 & {$[5,3,3,5,5,4,2,3]$} \\
\hline 30 & 2 & 6 & {$[4,4,3,1,5,2]$} \\
\hline
\end{tabular}

Table 3. Iterations and time taken by each method to find the weighted analytic center for the given weights using exact line search (Newton's method).

\begin{tabular}{|c|c|c|c|c|c|c|c|c|c|}
\hline \multirow{3}{*}{ Prob } & \multirow{3}{*}{$\begin{array}{l}\text { Weights } \\
\Omega\end{array}$} & \multirow{3}{*}{$\begin{array}{l}\text { FR } \\
\text { Iter }\end{array}$} & \multicolumn{3}{|c|}{ HS } & \multicolumn{2}{|l|}{ PR } & \multicolumn{2}{|c|}{ RAMI } \\
\hline & & & \multirow{2}{*}{$\begin{array}{l}\text { Time } \\
\text { (sec) }\end{array}$} & \multirow{2}{*}{ Iter } & \multirow{2}{*}{$\begin{array}{l}\text { Time } \\
\text { (sec) }\end{array}$} & \multirow{2}{*}{ Iter } & \multirow{2}{*}{$\begin{array}{l}\text { Time } \\
\text { (sec) }\end{array}$} & \multirow{2}{*}{ Iter } & \multirow{2}{*}{$\begin{array}{l}\text { Time } \\
\text { (sec) }\end{array}$} \\
\hline & & & & & & & & & \\
\hline 1 & {$[4,5]$} & 6 & 0.0053 & 4 & 0.0041 & 4 & 0.0033 & 5 & 0.0047 \\
\hline 2 & {$[3,175,1]$} & 14 & 0.0281 & 12 & 0.0228 & 11 & 0.0209 & 11 & 0.0219 \\
\hline 3 & {$[10,10,10,1,1,1,10,1]$} & 9 & 0.0416 & 8 & 0.0328 & 7 & 0.0295 & 9 & 0.0369 \\
\hline 4 & {$[100,1]$} & 31 & 0.0474 & 19 & 0.0281 & 16 & 0.0272 & 17 & 0.0249 \\
\hline 5 & {$[1,10]$} & 10 & 0.0154 & 8 & 0.0146 & 8 & 0.0123 & 9 & 0.0122 \\
\hline 6 & {$[1,1,100,100,100,1,100,10,1,1]$} & 5 & 0.2656 & 20 & 0.1203 & 17 & 0.0989 & 18 & 0.1059 \\
\hline 7 & {$[1,100,10,1,10,1,10]$} & 25 & 0.1045 & 16 & 0.0643 & 16 & 0.0674 & 19 & 0.0777 \\
\hline 8 & {$[10,10,1,10,1,1]$} & 42 & 0.1796 & 20 & 0.0839 & 28 & 0.1206 & 27 & 0.1142 \\
\hline
\end{tabular}




\begin{tabular}{|c|c|c|c|c|c|c|c|c|c|}
\hline \multirow{3}{*}{ Prob } & \multirow{3}{*}{$\begin{array}{l}\text { Weights } \\
\Omega\end{array}$} & \multicolumn{2}{|l|}{$\mathbf{F R}$} & \multicolumn{2}{|l|}{ HS } & \multicolumn{2}{|l|}{ PR } & \multicolumn{2}{|c|}{ RAMI } \\
\hline & & \multirow{2}{*}{ Iter } & \multirow{2}{*}{$\begin{array}{l}\text { Time } \\
\text { (sec) }\end{array}$} & \multirow{2}{*}{ Iter } & \multirow{2}{*}{$\begin{array}{l}\text { Time } \\
\text { (sec) }\end{array}$} & \multirow{2}{*}{ Iter } & \multirow{2}{*}{$\begin{array}{l}\text { Time } \\
\text { (sec) }\end{array}$} & \multirow{2}{*}{ Iter } & \multirow{2}{*}{$\begin{array}{l}\text { Time } \\
\text { (sec) }\end{array}$} \\
\hline & & & & & & & & & \\
\hline 9 & {$[100,1,1,1]$} & 107 & 0.2857 & 49 & 0.1328 & 48 & 0.1304 & 137 & 0.3728 \\
\hline 10 & {$[10,1,100]$} & 20 & 0.0517 & 19 & 0.0444 & 21 & 0.0482 & 23 & 0.0537 \\
\hline 11 & {$[1,1,1,10,10,1,100,1]$} & 46 & 0.2458 & 25 & 0.1315 & 24 & 0.1257 & 31 & 0.1646 \\
\hline 12 & {$[1,10]$} & 47 & 0.0912 & 36 & 0.0682 & 34 & 0.0644 & 53 & 0.1011 \\
\hline 13 & {$[1,10,1,100]$} & 52 & 0.1467 & 35 & 0.0989 & 38 & 0.1063 & 64 & 0.1797 \\
\hline 14 & {$[1,1,10,10,10]$} & 34 & 0.1317 & 34 & 0.1321 & 35 & 0.1314 & 42 & 0.1594 \\
\hline 15 & {$[10,10,1,1,100]$} & 90 & 0.3723 & 37 & 0.1528 & 24 & 0.1006 & 49 & 0.2001 \\
\hline 16 & {$[10,1,1]$} & 209 & 0.5898 & 73 & 0.2014 & 71 & 0.2052 & 268 & 0.750 \\
\hline 17 & {$[100,100,1]$} & 70 & 0.1941 & 56 & 0.1546 & 41 & 0.1146 & 76 & 0.2110 \\
\hline 18 & {$[10,1,1,1,10,10,1,1]$} & 48 & 0.3132 & 27 & 0.1725 & 29 & 0.1874 & 30 & 0.1951 \\
\hline 19 & {$[1,10,1,100,1,10,10,1]$} & 177 & 1.1595 & 33 & 0.2144 & 33 & 0.2177 & 46 & 0.3010 \\
\hline 20 & {$[1,10,10,1,1,1,1,1,100]$} & 85 & 0.6547 & 38 & 0.2885 & 46 & 0.3509 & 61 & 0.4712 \\
\hline 21 & {$[1,1,1,1,10,1]$} & 38 & 0.1313 & 16 & 0.0538 & 11 & 0.0385 & 11 & 0.0366 \\
\hline 22 & {$[10,1,1,1,10,1,1]$} & 18 & 0.0801 & 15 & 0.0659 & 13 & 0.0642 & 16 & 0.0679 \\
\hline 23 & {$[100,1,1]$} & 22 & 0.0479 & 27 & 0.0617 & 27 & 0.0621 & 25 & 0.0569 \\
\hline 24 & {$[100,1,100,100,100,10,1,1,10]$} & 35 & 0.2086 & 20 & 0.1149 & 19 & 0.1089 & 23 & 0.1306 \\
\hline 25 & {$[1,100,10]$} & 61 & 0.1757 & 65 & 0.1887 & 59 & 0.1707 & 132 & 0.3827 \\
\hline 26 & {$\left[7,7,7,8,7,6,4,10^{6}, 3,6\right]$} & 87 & 0.6418 & 92 & 0.6529 & 79 & 0.5501 & 177 & 1.2600 \\
\hline 27 & {$\left[3,5,2,1,10^{6}, 2,7\right]$} & 43 & 0.2032 & 37 & 0.1607 & 32 & 0.1516 & 53 & 0.2383 \\
\hline 28 & {$\left[1,1,4,8,5,8,3,10^{6}\right]$} & 42 & 0.2234 & 35 & 0.1906 & 37 & 0.2048 & 32 & 0.1697 \\
\hline 29 & {$\left[2,10^{6}, 4,3\right]$} & 32 & 0.0952 & 33 & 0.0919 & 18 & 0.0535 & 29 & 0.0802 \\
\hline 30 & {$\left[4,6,5,3,10^{6}, 4\right]$} & 13 & 0.0464 & 8 & 0.0267 & 8 & 0.0315 & 9 & 0.0291 \\
\hline
\end{tabular}

Table 4. Iterations and time taken by each method to find the weighted analytic center for the given weights using inexact line search (Quadratic Interpolation).

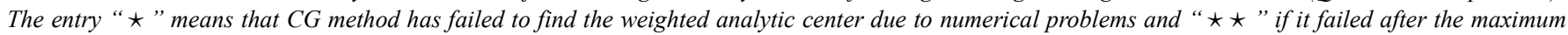
number of 1000 iterations (jamming phenomenon).

\begin{tabular}{|c|c|c|c|c|c|c|c|c|c|}
\hline \multirow{3}{*}{ Prob } & \multirow{3}{*}{$\begin{array}{l}\text { Weights } \\
\Omega\end{array}$} & \multirow{3}{*}{$\begin{array}{l}\text { FR } \\
\text { Iter }\end{array}$} & \multirow{3}{*}{$\begin{array}{l}\text { Time } \\
\text { (sec) }\end{array}$} & \multirow{3}{*}{$\begin{array}{l}\text { HS } \\
\text { Iter } \\
\end{array}$} & \multirow{3}{*}{$\begin{array}{l}\text { Time } \\
\text { (sec) }\end{array}$} & \multirow{3}{*}{$\begin{array}{l}\text { PR } \\
\text { Iter }\end{array}$} & \multicolumn{3}{|c|}{ RAMI } \\
\hline & & & & & & & \multirow{2}{*}{$\begin{array}{l}\text { Time } \\
\text { (sec) } \\
\end{array}$} & \multirow{2}{*}{ Iter } & \multirow{2}{*}{$\begin{array}{l}\text { Time } \\
\text { (sec) } \\
\end{array}$} \\
\hline & & & & & & & & & \\
\hline 1 & {$[4,5]$} & 5 & 0.0065 & 5 & 0.0076 & 5 & 0.0069 & 6 & 0.0090 \\
\hline 2 & {$[3,175,1]$} & 29 & 0.0783 & 12 & 0.0266 & 9 & 0.0211 & 10 & 0.0229 \\
\hline 3 & {$[10,10,10,1,1,1,10,1]$} & 10 & 0.0546 & 7 & 0.0342 & 6 & 0.0277 & 7 & 0.0349 \\
\hline 4 & {$[100,1]$} & 19 & 0.0367 & 13 & 0.0219 & 15 & 0.0258 & 20 & 0.0341 \\
\hline 5 & {$[1,10]$} & 12 & 0.0229 & 10 & 0.0179 & 10 & 0.0185 & 11 & 0.0171 \\
\hline 6 & {$[1,1,100,100,100,1,100,10,1,1]$} & 19 & 0.1283 & 20 & 0.1349 & 19 & 0.1274 & 20 & 0.1386 \\
\hline 7 & {$[1,100,10,1,10,1,10]$} & 19 & 0.0942 & 14 & 0.0687 & 13 & 0.0632 & 19 & 0.1054 \\
\hline 8 & {$[10,10,1,10,1,1]$} & 30 & 0.1526 & 29 & 0.1411 & 29 & 0.1391 & 30 & 0.1454 \\
\hline 9 & {$[100,1,1,1]$} & 242 & 0.7563 & 32 & 0.0963 & 29 & 0.0871 & 31 & 0.0939 \\
\hline 10 & {$[10,1,100]$} & 20 & 0.0568 & 17 & 0.0466 & 18 & 0.0497 & 22 & 0.0601 \\
\hline 11 & {$[1,1,1,10,10,1,100,1]$} & 61 & 0.3749 & 33 & 0.2000 & 26 & 0.1590 & 28 & 0.1711 \\
\hline 12 & {$[1,10]$} & 123 & 0.2597 & 35 & 0.0699 & 34 & 0.0687 & 51 & 0.1018 \\
\hline 13 & {$[1,10,1,100]$} & 59 & 0.1865 & 36 & 0.1122 & 32 & 0.0992 & 67 & 0.2088 \\
\hline 14 & {$[1,1,10,10,10]$} & 34 & 0.1425 & 33 & 0.1387 & 33 & 0.1390 & 41 & 0.1700 \\
\hline 15 & {$[10,10,1,1,100]$} & 92 & 0.4288 & 74 & 0.3430 & 30 & 0.1354 & 43 & 0.1967 \\
\hline 16 & {$[10,1,1]$} & 87 & 0.2431 & 93 & 0.2573 & 70 & 0.1995 & 229 & 0.6478 \\
\hline 17 & {$[100,100,1]$} & 47 & 0.1504 & 45 & 0.1385 & 46 & 0.1536 & 71 & 0.2179 \\
\hline 18 & {$[10,1,1,1,10,10,1,1]$} & 26 & 0.1847 & 29 & 0.2082 & 28 & 0.1982 & 29 & 0.2057 \\
\hline 19 & {$[1,10,1,100,1,10,10,1]$} & 108 & 0.7878 & 39 & 0.2825 & 37 & 0.2677 & 45 & 0.3293 \\
\hline 20 & {$[1,10,10,1,1,1,1,1,100]$} & 117 & 0.9866 & 45 & 0.3787 & 44 & 0.3703 & 59 & 0.5027 \\
\hline 21 & {$[1,1,1,1,10,1]$} & 10 & 0.0420 & 9 & 0.0333 & 11 & 0.0417 & 9 & 0.0355 \\
\hline 22 & {$[10,1,1,1,10,1,1]$} & 35 & 0.1763 & 18 & 0.0877 & 15 & 0.0715 & 15 & 0.0719 \\
\hline 23 & {$[100,1,1]$} & 22 & 0.0565 & 28 & 0.0710 & 28 & 0.0706 & 25 & 0.0633 \\
\hline 24 & {$[100,1,100,100,100,10,1,1,10]$} & 27 & 0.1853 & 22 & 0.1452 & 19 & 0.1228 & 24 & 0.1583 \\
\hline 25 & {$[1,100,10]$} & 64 & 0.2045 & 59 & 0.1834 & 66 & 0.2108 & 130 & 0.4091 \\
\hline 26 & {$\left[7,7,7,8,7,6,4,10^{6}, 3,6\right]$} & $* *$ & $* *$ & $*$ & $*$ & $*$ & $*$ & $*$ & $*$ \\
\hline 27 & {$\left[3,5,2,1,10^{6}, 2,7\right]$} & ** & $* *$ & $*$ & $*$ & ** & $* *$ & $*$ & $*$ \\
\hline 28 & {$\left[1,1,4,8,5,8,3,10^{6}\right]$} & $*$ & $*$ & $*$ & $*$ & $*$ & $*$ & $*$ & $*$ \\
\hline 29 & {$\left[2,10^{6}, 4,3\right]$} & $* *$ & $* *$ & $*$ & $*$ & $*$ & $*$ & $*$ & $*$ \\
\hline 30 & {$\left[4,6,5,3,10^{6}, 4\right]$} & $*$ & $*$ & $*$ & $*$ & $*$ & $*$ & $*$ & $*$ \\
\hline
\end{tabular}




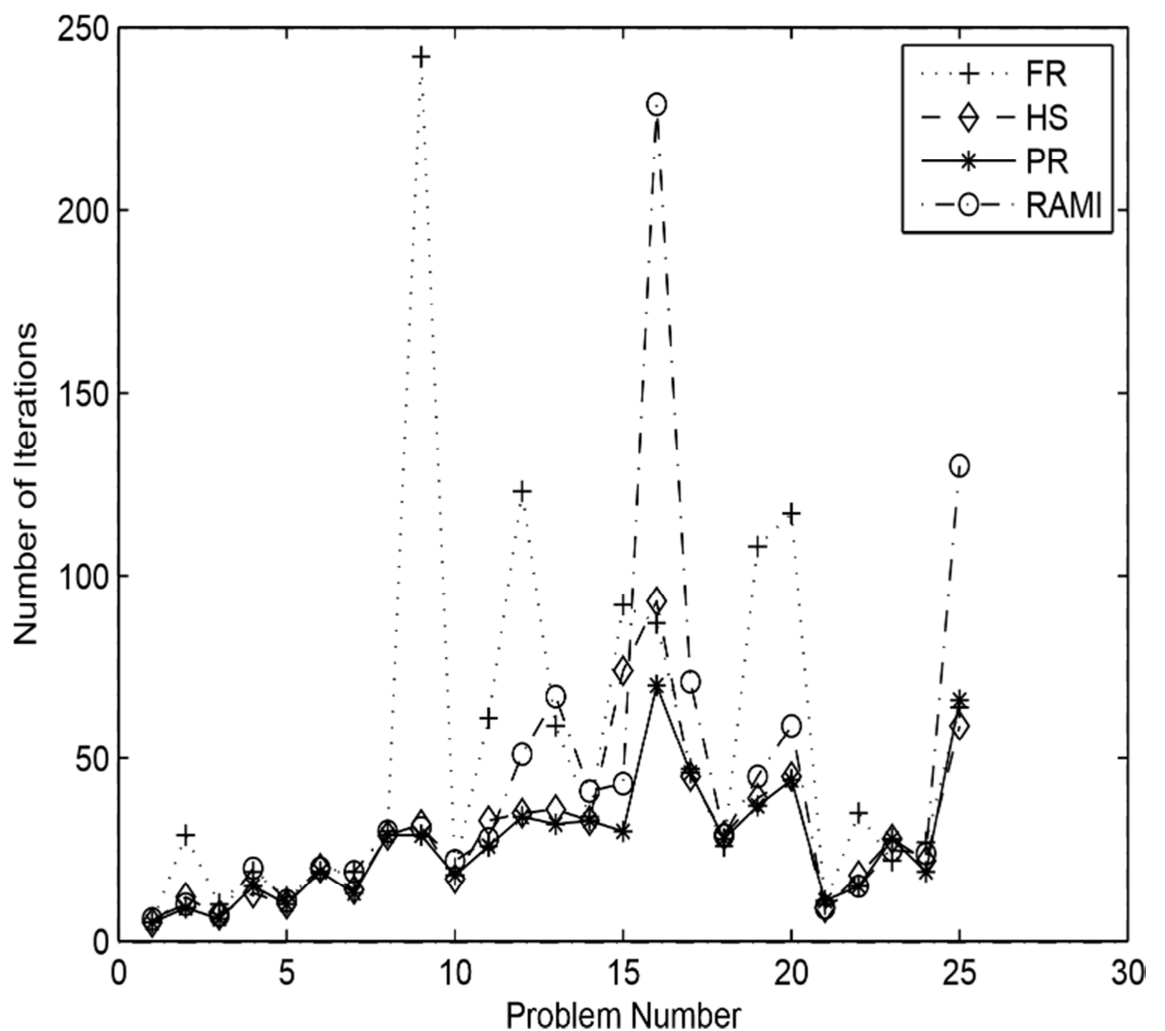

Figure 1. Problem Number Vs Iterations taken by each method to find the weighted analytic center using exact line search (Newton's method), where $+=$ FR, $=H S$, *=PR, $o=R A M I$.

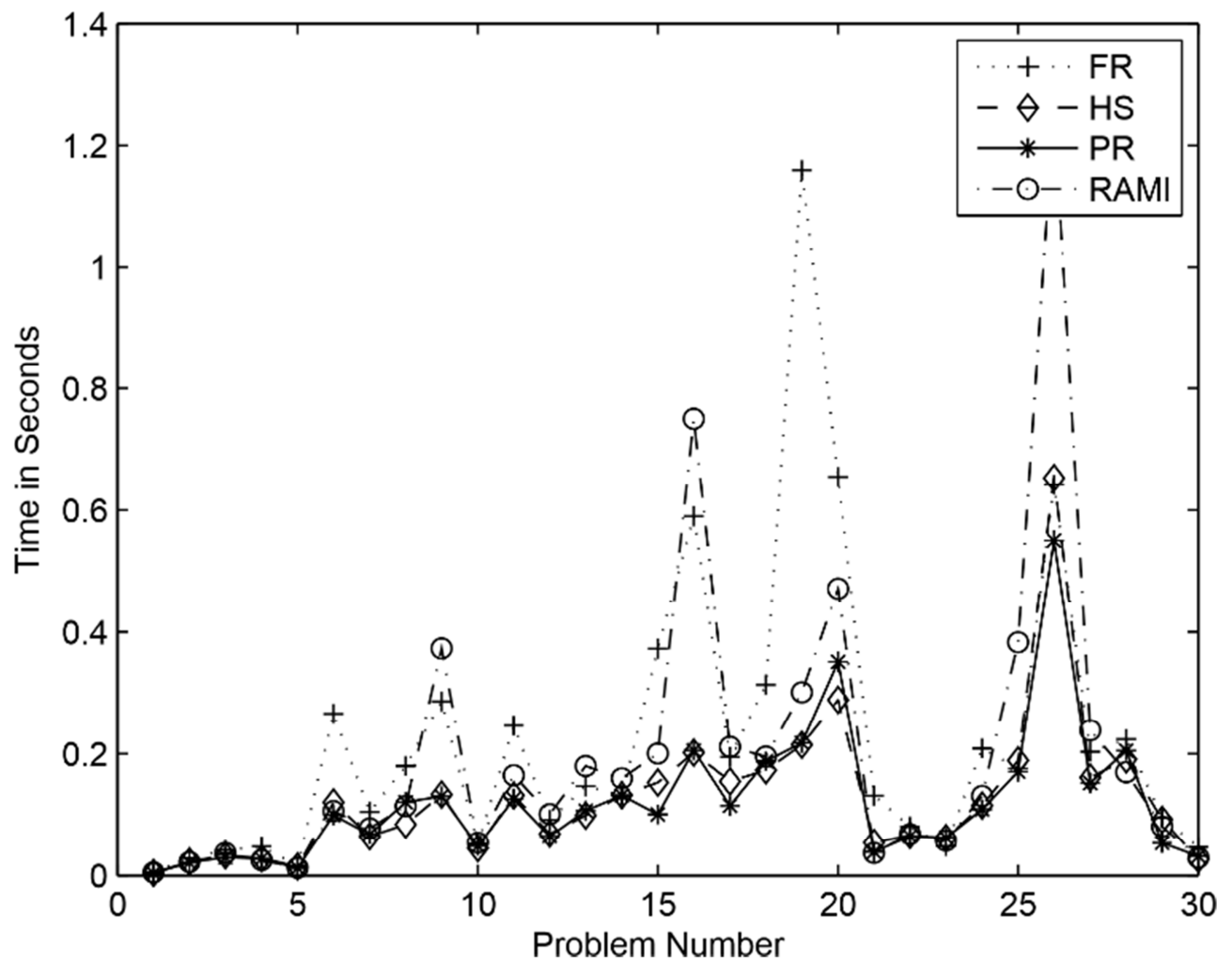

Figure 2. Problem Number Vs Time taken by each method to find the weighted analytic center using exact line search (Newton's method), where $+=$ FR, =HS, $*=P R, o=R A M I$. 

Linear Matrix Inequalities Using Exact and Quadratic Interpolation Line Searches

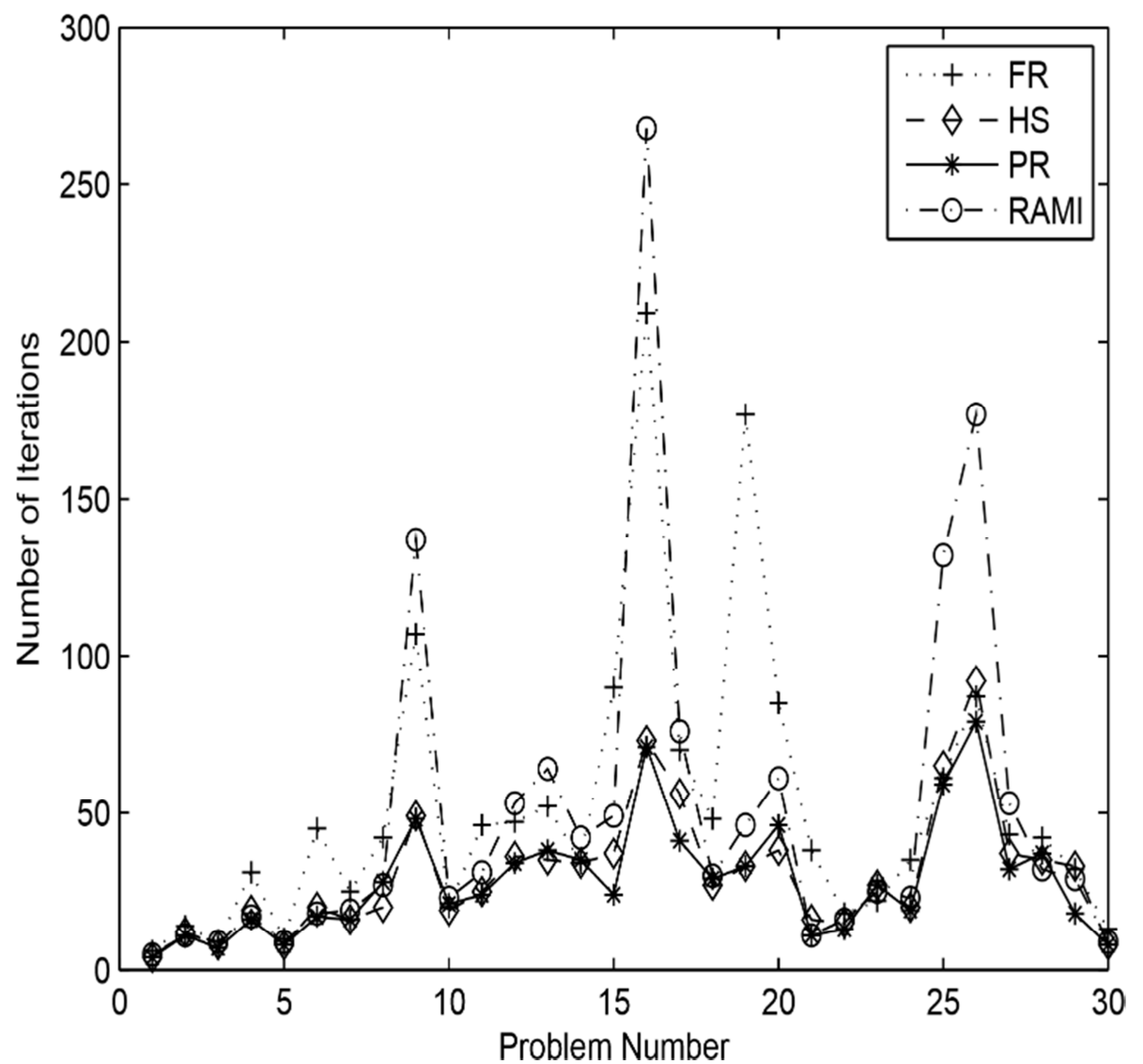

Figure 3. Problem Number Vs Iterations taken by each method to find the weighted analytic center using inexact line search (Quadratic Interpolation) for the 25 problems where all four methods were successful and $+=F R,=H S, *=P R, o=R A M I$.

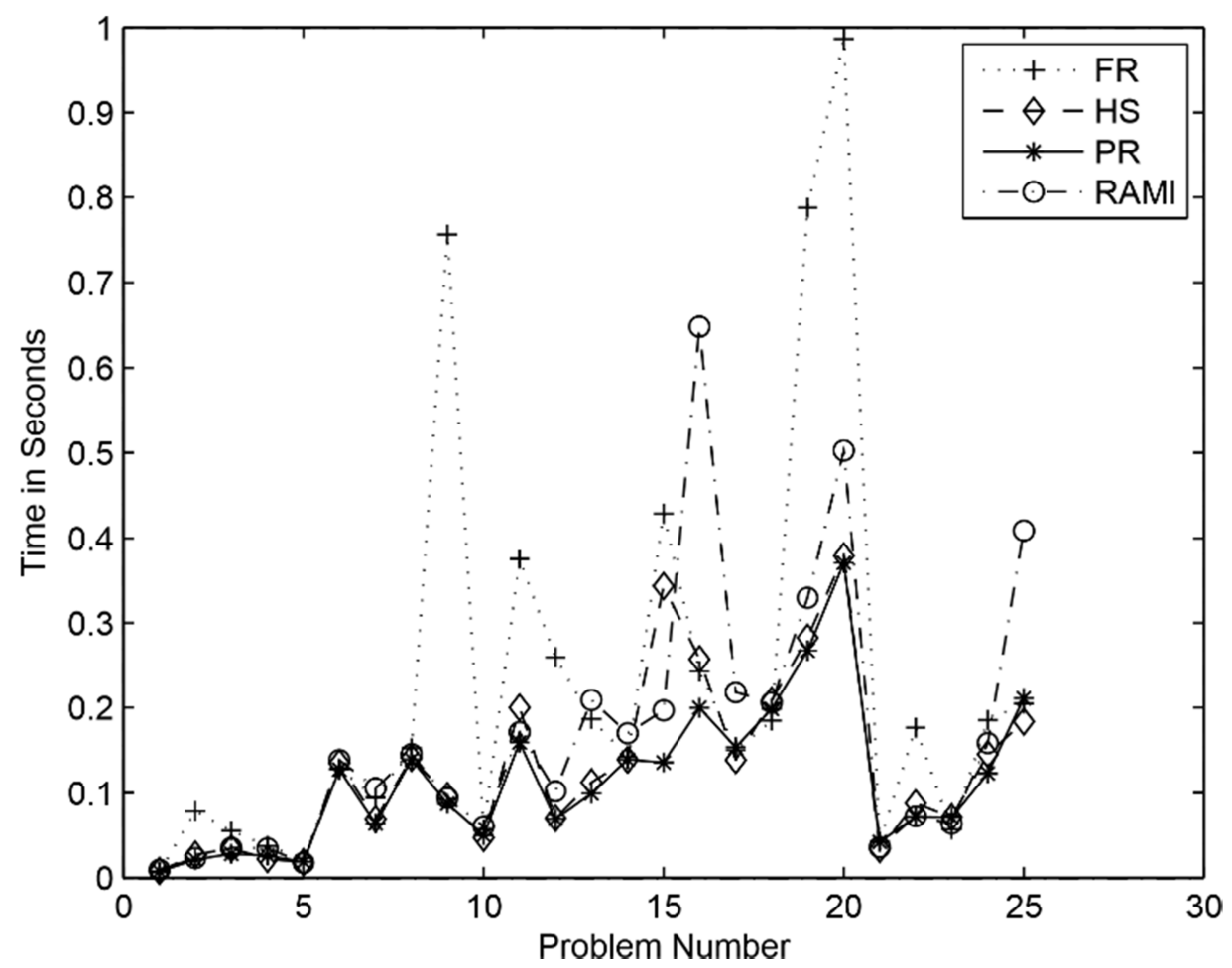

Figure 4. Problem Number Vs Time taken by each method to find the weighted analytic center using inexact line search (Quadratic Interpolation) for the 25 problems where all four methods were successful and $+=F R,=H S, *=P R, o=R A M I$. 


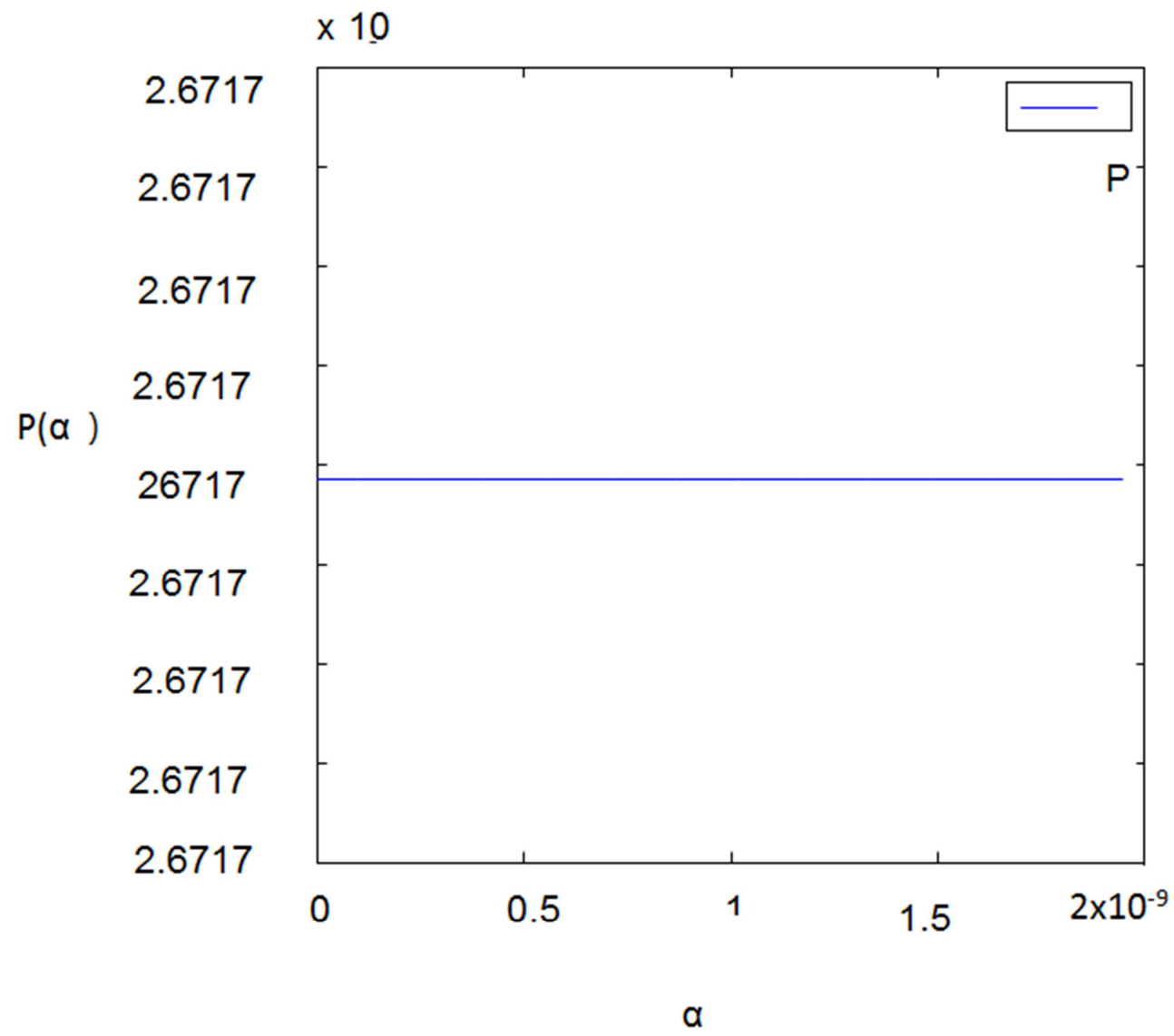

Figure 5. Graph of the Quadratic Approximation $P(\alpha)$ for Problem 30 with $w=\left[4,6,5,3,10^{6}, 4\right]$ at iteration 12. Note $P(\alpha)$ is flat over the interval $\left[h_{1}, h_{3}\right]=[0$, $1.9462 \times 10^{-9} \mathrm{~J}$. At iteration 13, $P(\alpha)$ does not exist and therefore $F R$ with Quadratic interpolation line search failed.

The graph of Problem Number vs. Number of Iterations for Table 3 is given in Figure 1 and Figure 2 is the graph of the Problem Number vs. Time taken. The results in Table 3, Figure 1 and Figure 2 show that PR is the best method in terms of least number of iterations. Table 4 gives the number of iterations and time (in seconds) taken by each method to find the weighted analytic center for the given weights using Quadratic interpolation inexact line search. The entry " $\star$ " means that CG method has failed to find the weighted analytic center due to numerical problems and " $\star \star$ " if it did not converge after the maximum number of 1000 iterations (jamming phenomenon). We see that FR had jamming in three problems, while PR had jamming in one problem and HS in none. This confirms the known fact that FR is more susceptible to jamming than both PR and HS. Figure 3 is the graph of the Problem Number vs. Number of Iterations taken by each method to find the weighted analytic center using inexact line search (Quadratic Interpolation) for the 25 problems where all four methods were successful. The graph of Problem Number vs Time taken is given in Figure 4. The results in Table 4, Figure 3 and Figure 4 show that PR is the best method in terms of least number of iterations and time, followed by HS, then RAMI, and then FR.

Therefore, FR with Quadratic interpolation line search failed for Problem 30. Our results show that PR and HS are superior to RAMI with the problems considered in this paper, contrary to the results reported in [23].

\section{Conclusion}

We have studied four conjugate gradient algorithms applied to the problem of weighted analytic center for linear matrix inequalities. The methods considered are HS, FR, PR and RAMI.

For each method, we consider exact line search and Quadratic interpolation line search. We use numerical experiments on randomly generated test problems to compare performance of each method by looking at the number of iterations and time taken to compute the weighted analytic center. We use one-dimensional Newton's method exact line search and Quadratic Interpolation inexact line search. Our numerical results show that PR is the best method, followed by HS, then RAMI, and then FR. We find that PR performed nearly the same as HS with exact line search, which confirmed what is known in the literature. They also show that both line searches work well, but exact line search handles weights better than the inexact Quadratic interpolation line search when some weight is relatively much larger than the other weights. We find that all the CG methods with Quadratic interpolation inexact line search failed on each problem where some weight is relatively much larger than the remaining weights. We intend to investigate the same problem using hybrid conjugate gradient methods in another paper. We are not aware of any convergence analysis for FR, HS, PR or RAMI with Quadratic interpolation inexact line search, we hope to study it in the future. 


\section{Acknowledgements}

We thank Lieven Vandenberghe of University of California at Los Angeles and Jim Swift of Northern Arizona University for responding to our questions during this research.

\section{References}

[1] F. Alizadeh, "Interior Point Methods in Semidefinite Programming with Applications to Combinatorial Optimization”, SIAM Journal on Optimization, vol. 5, no. 1, 1995, pp. 13-51.

[2] L. Vandenberghe and S. Boyd, "Semidefinite Programming", SIAM Review, vol. 38, 1996, pp. 49-95.

[3] F. Alizadeh, J. A. Haeberly and M. Overton, "Primal-Dual Methods for Semidefinite Programming: Convergence Rates, Stability and Numerical Results", SIAM Journal on Optimization, vol. 8, no. 3, 1988, pp. 746-768.

[4] J. Renegar, "A polynomial-time algorithm, based on Newton's method, for linear programming, " Math. Programming, vol. 40, 1988, pp. 59-93.

[5] M. J. Todd, K. C. Toh and R. H. Tuntuncu, "On the Nesterov-Todd direction in semidefinite programming," SIAM J. Optim., vol. 8, 1998, pp. 769-796.

[6] L. Vandenberghe, S.-P. Boyd and S. Wu, "Determinant Maximization with Linear Matrix Inequality Constraints", SIAM Journal on Matrix Analysis, vol. 19, no. 2, 1998, pp. 499-533.

[7] I. S. Pressman and S. Jibrin, "A Weighted Analytic Center for Linear Matrix Inequalities", Journal of Inequalities in Pure and Applied Mathematics, Vol. 2, Issue 3, Article 29, 2002.

[8] S. Jibrin and J. W. Swift, "The Boundary of the Weighted Analytic Center for Linear Matrix inequalities." Journal of Inequalities in Pure and Applied Mathematics, Vol. 5, Issue 1, Article 14, 2004.

[9] J. Machacek and S. Jibrin, "An Interior Point Method for Solving Semidefinite Programs Using Cutting Planes and Weighted Analytic Centers", Journal of Applied Mathematics, Vol. 2012, Article ID 946893, 21 pages.

[10] D. S. Atkinson and P. M. Vaidya, "A scaling technique for finding the weighted analytic center of a polytope "Math. Prog., vol. 57, 1992, pp. 163-192.

[11] S. Jibrin, "Computing Weighted Analytic Center for Linear Matrix Inequalities Using Infeasible Newton's Method", Journal of Mathematics, vol. 2015, Article ID 456392, 2015.

[12] S. Jibrin, I. Abdullahi, "Search Directions in Infeasible Newton's method for Computing Weighted Analytic Center for Linear Matrix Inequalities", Applied and Computational Mathematics, vol. 8, no. 1, 2019, pp. 21-28.

[13] L. Vandenberghe and S. Boyd, "Convex Optimization", Cambridge University Press, New York 2004.

[14] W. W. Hager, H. Zhang, "A survey of nonlinear conjugate gradient method", Pacific Journal of Optimization, vol. 2, no. 1, 2006, pp. 35-58.

[15] M. Rivaie, A. Abashar, M. Mamat and M. Ismail, "The convergence properties of a new type of conjugate gradient methods", Applied Mathematical Sciences, vol. 9, no. 54, 2016, pp. 2671-2682.

[16] I. Abdullahi, R. Ahmad, "Convergence Analysis of a New Conjugate Gradient Method for Unconstrained Optimization", Applied Mathematical Sciences, vol. 9, no. 140, 2015, pp. 6969-6984.

[17] I. Abdullahi, R. Ahmad, "Global Convergence Analysis of a Nonlinear Conjugate Gradient Method for Unconstrained Optimization Problems", Indian Journal of Science and Technology, vol. 9, no. 41, DOI: 10.17485/ijst/2016/v9i41/90175, 2016.

[18] I. Abdullahi, R. Ahmad, "Global convergence analysis of a new hybrid conjugate gradient method for unconstrained optimization problems", Malaysian Journal of Fundamental and Applied Sciences, vol. 2, no. 13, 2017, pp. 40-48.

[19] X. Du, J. Liu, "Global convergence of a spectral hs conjugate gradient method", Procedia Engineering, vol. 15, 2011, pp. 1487-1492.

[20] W. W. Hager, H. Zhang, "A new conjugate gradient method with guaranteed descent and an efficient line search", JSIAM Journal on Optimization, vol. 16, no. 1, 2005, pp. 170-192.

[21] J. Liu, Y. Feng, "Global convergence of a modified ls nonlinear conjugate gradient method", Procedia Engineering, vol. 15, 2011, pp. 4357-4361.

[22] Z. Wei, G. Li, L. Qi, "New nonlinear conjugate gradient formulas for large-scale unconstrained optimization problems", Applied Mathematics and Computation, vol. 179, no. 2, 2006, pp. 407-430.

[23] Y. Zhang, H. Zheng, C. Zhang, "Global convergence of a modified PRP conjugate gradient method", Procedia Engineering, vol. 31, 2012, pp. 986-995.

[24] R. Fletcher, C. M. Reeves, "Function minimization by conjugate gradients", The computer Journal, vol. 7, no. 2, 1964, pp. $149-154$.

[25] B. T. Polyak, "The conjugate gradient method in extremal problems", USSR Computational Mathematics and Mathematical Physics, vol. 9, no. 4, 1969, pp. 94-112.

[26] M. R. Hestenes, E. Stiefel, "Methods of conjugate gradients for solving linear systems", Journal of Research of the National Bureau of Standards, vol. 49, 1952, pp. 409-436.

[27] S. Jibrin and I. S. Pressman, "Monte Carlo Algorithms for the Detection of Necessary Linear Matrix Inequalities", International Journal of Nonlinear Sciences and Numerical Simulation, vol. 2, no. 2, 2001. pp. 139-154.

[28] R. Burden and D. Faires, "Numerical Analysis", 9th Ed., Addison Wesley, 2011.

[29] G. Zoutendijk, Nonlinear Programming, Computational Methods, in Integer and Nonlinear Programming, J. Abadie, ed., North-Holland, Amsterdam, 1970, pp. 37-86.

[30] E. Polak and G. Ribiere, "Note sur la convergence de directions conjugees", Rev. Francaise Informat Recherche Opertionelle, 3e Annee 16, 1969, pp. 35-43.

[31] M. J. D. Powell, "Restart procedures of the conjugate gradient method", Math. Prog., vol. 2, 1977, pp. 241-254. 